\title{
Variables related to increased mortality following out-patient pulmonary rehabilitation
}

\author{
D.A. Gerardi, L. Lovett, M.L. Benoit-Connors, J.Z. Reardon, R.L. ZuWallack
}

\begin{abstract}
Variables related to increased mortality following out-patient pulmonary rehabilitation. D.A. Gerardi, L. Lovett, M.L. Benoit-Connors, J.Z. Reardon, R.L. ZuWallack. (CERS Journals Ltd. 1996.

ABSTRACT: Although patients with advanced pulmonary disease have significant improvement in exercise ability and functional status following comprehensive outpatient pulmonary rehabilitation (OPR), their long-term prognosis once they have reached this stage of their diseases remains poor.

To further evaluate predictors of increased mortality in these patients, we related patient characteristics and short-term outcome obtained during OPR assessment of 158 patients to subsequent survival. The time period from OPR to death or collection of survival data was $\mathbf{4 0 . 0} \pm \mathbf{1 7 . 1}$ months. The following variables were tested individually and in stepwise fashion using a proportional hazards model: 1) age; 2) gender; 3) pulmonary diagnosis; 4) prebronchodilator forced expiratory volume in one second (FEV1); 5) arterial oxygen tension $\left(\mathrm{Pa}_{\mathrm{a}} \mathrm{O}_{2}\right)$ and arterial carbon dioxide tension $\left(\mathrm{Pa}_{\mathrm{a}}, \mathrm{CO}_{2}\right)$; 6) body mass index $(\mathrm{BMI})$; 7) pre- and post-OPR $12 \mathrm{~min}$ walking distance (12-MW); 8) pre- and post-OPR quality of life, using the Chronic Respiratory Disease Questionnaire (CRDQ); 9) number and type of nonpulmonary diagnoses; and 10) number of medications. Separate survival analyses were performed for all deaths (the total group), respiratory deaths only (nonrespiratory deaths excluded), and nonrespiratory deaths only (respiratory deaths excluded).

Forty three patients $(27 \%)$ died during the study period; and the 3 year survival was $80 \%$. For all three survival analyses, the post-OPR 12-MW was the most significant variable related to prognosis: patients with low timed walking distance had increased mortality both from respiratory and nonrespiratory causes. Other variables related to increased mortality included: elevated $\mathrm{Pa}_{\mathrm{a}} \mathrm{CO}_{2}$; low pre-OPR 12-MW; reduced $\mathrm{Pa}_{\mathrm{a}} \mathrm{O}_{2}$; low $\mathrm{FEV} 1$; low BMI, increased number of medications, and increased CRDQ dyspnoea.

These results indicate that the timed walking distance following out-patient pulmonary rehabilitation is an important predictor of survival in patients with advanced pulmonary disease.
\end{abstract}

Eur Respir J., 1996, 9, 431-435.

Rehabilitation of advanced chronic lung disease frequently leads to significant improvement in exercise ability and quality of life [1]. Despite gains in functional status, the mortality of individuals reaching this stage of their disease remains high $[2,3]$. Variables found to be associated with a poor prognosis include: advanced age; low forced expiratory volume in one second (FEV1); reduced diffusing capacity; elevated resting or exercise heart rate; decreased body weight; reduced serum albumin; hypoxaemia or hypercarbia; right ventricular disease; decreased exercise tolerance; and reduced quality of life or performance status [4-8]. Although thorough patient evaluation and multidimensional outcome measurement are routine in many pulmonary rehabilitation programmes, the relationship between results from this assessment and subsequent survival has not been investigated. This study evaluated the relationship between patient variables recorded
University of Connecticut School of Medicine, Farmington, CT, USA and Section of Pulmonary Diseases, Saint Francis Hospital and Medical Center, Hartford, CT, USA

Correspondence: D. Gerardi Section of Pulmonary Diseases Saint Francis Hospital and Medical Center 114 Woodland Street

Hartford

CT 06105

USA

Keywords: Mortality rehabilitation

timed walking distance

Received: February 281995

Accepted after revision November 41995 at pulmonary rehabilitation assessment to subsequent prognosis.

\section{Methods}

\section{Patients and pulmonary rehabilitation}

The relationship between variables recorded during routine patient assessment for out-patient pulmonary rehabilitation (OPR) and subsequent survival was retrospectively analysed. Patients completing our OPR programme between November 1989 and March 1993 were included in the analysis. OPR referrals were most often made by an internist or pulmonary specialist, usually following a gradual deterioration in the patient's respiratory status. All patients had significant respiratory 
symptoms despite usual medical therapy. Active cigarette smokers were not eligible for OPR.

A clinical assessment, including listing of diagnoses and medications, was performed by the pulmonary rehabilitation nurse prior to rehabilitation. Prebronchodilator spirometry was routinely performed prior to rehabilitation. Arterial blood gas determinations, while not required for the programme, were available in most patients. The 12 min walking distance (12-MW) [9] was used as a primary outcome measure for OPR and obtained before and shortly following OPR. Beginning in 1990, the Chronic Respiratory Disease Questionnaire (CRDQ) [10], a respiratory-specific quality of life instrument, was also used as a pre- post-OPR outcome measure.

The OPR programme consisted of $3 \mathrm{~h}$ sessions held twice weekly for 6 weeks. Approximately half the time spent in each session was educational, with topics including symptom management, medications, compliance, breathing retraining, pacing, nutrition, and stress reduction. The remainder of the time was spent on exercise conditioning. Exercise included upper extremity training with weights and elastic bands, inspiratory resistive exercise, and lower extremity training with a treadmill and stationary bicycle. From four to eight patients were enrolled in each 6 week block. Eight blocks were held each year.

\section{Predictor variables}

Records from the 158 patients who completed the OPR programme between November 1989 and March 1993 were reviewed. From this review, the following variables were analysed:

1. Age, in years.

2. Gender.

3. Pulmonary diagnosis. This was defined as the principal pulmonary disorder that led to the OPR referral. To simplify the analysis, the pulmonary diagnosis was classified as either: a) chronic obstructive pulmonary disease (COPD); b) asthma; c) restrictive disease (including chest wall disorders and pulmonary fibrosis); or d) bronchiectasis (including cystic fibrosis). Patients with asthmatic bronchitis were placed into the COPD category.

4. Prebronchodilator FEV1. This measurement was available in 147 patients. Postbronchodilator FEV1 was not routinely performed.

5. Arterial oxygen tension $\left(\mathrm{Pa}_{\mathrm{a}} \mathrm{O}_{2}\right)$ and arterial carbon dioxide tension $\left(\mathrm{Pa}_{\mathrm{a}} \mathrm{CO}_{2}\right)$. Room air arterial blood gases were obtained in 130 patients prior to OPR.

6. Weight, expressed as body mass index (BMI) $\left(\mathrm{kg} \cdot \mathrm{m}^{-2}\right)$. Patients with a BMI $<20 \mathrm{~kg} \cdot \mathrm{m}^{-2}$ were considered underweight; $20-25 \mathrm{~kg} \cdot \mathrm{m}^{-2}$ normal; and $>25 \mathrm{~kg} \cdot \mathrm{m}^{-2}$ overweight. 7 . The pre- and post-OPR 12-MW. The pre-OPR 12MW was measured in 149 patients and the post-OPR 12MW in 147. Pre- to post-OPR change in 12-MW was analysed separately.

8. Pre- and post-OPR CRDQ quality of life. This 20 item instrument provides a total score and dimension subscores of dyspnoea, function, emotion, and mastery (the feeling of control over the disease). Pre-OPR CRDQ data were available in 114 patients and post-OPR data in 112. Pre- to post-OPR change in quality of life was analysed separately.

9. Nonpulmonary diagnoses. These were included in the present analysis if they: a) caused symptoms; b) required regular medical or presciption therapy; or c) were of major clinical significance (such as a history of cancer or myocardial infarction). Skin diseases (other than melanoma), eye diseases, and obesity were not included in the analysis. The total number of nonpulmonary diagnoses and individual co-morbid conditions were analysed separately.

10. Medications. With the exception of regular use of oral antacids for acid-peptic disease, only prescription medications were analysed. Topical eye and skin medications were not analysed. When the same patient used both metered-dose and nebulized beta-agonists, this was considered as one drug.

\section{Survival}

Survival data, which were available for all 158 patients, were obtained from pulmonary rehabilitation records, physicians offices, correspondence with patients and their families, hospital records, and death certificates. The time period, in months, from the end of OPR to either the patient's death (for nonsurvivors) or collection of survival data (for survivors) was used for survival analysis. The post-OPR study period was $40.0 \pm 17.1$ months, and ranged 20-60 months. Deaths were categorized as either respiratory or nonrespiratory. Of the 41 deaths in this period, 26 were considered as primarily due to respiratory disease and 15 as nonrespiratory causes.

Three separate survival analyses were performed:

1. All deaths. Survivors, respiratory and nonrespiratory deaths were analysed $(n=158)$.

2. Respiratory deaths only. For this, the 15 nonrespiratory deaths were excluded; only survivors and respiratory deaths were analysed $(n=143)$.

3. Nonrespiratory deaths only. For this, the 26 respiratory deaths were excluded; only survivors and nonrespiratory deaths were analysed $(n=132)$.

\section{Data analysis}

Continuous variables were expressed as mean \pm SD, whilst categorical variables were expressed as percentages of the group total. Pearson correlations (r) were used to evaluate relationships between pre-OPR variables. The Cox proportional hazards model [11] was used for survival analysis. For this analysis time (in months) from post-OPR to either death or the current analysis was the response variable and survival - nonsurvival was the censoring variable. Survival analyses were performed individually for each predictor variable. In addition, all predictor variables found to be significant in individual analyses were included in a forward stepwise model. Separate survival analyses were performed for the entire group, respiratory deaths, and nonrespiratory deaths. A p-value of less than 0.05 was considered significant. 
Table 1. - Patient characteristics

\begin{tabular}{lcccc}
\hline Variable & Mean & SD & Minimum & Maximum \\
\hline Age yrs & 67 & 10 & 25 & 89 \\
Smoking pack-yrs & 50 & 31 & 0 & 180 \\
BMI kg· m-2 & 24.3 & 4.9 & 14.6 & 43.2 \\
FEV1 L & 1.03 & 0.56 & 0.25 & 3.31 \\
NP diagnoses n & 2.0 & 1.6 & 0 & 9 \\
Medications n & 5.8 & 2.7 & 0 & 15 \\
Pre-OPR 12-MW m & 659 & 223 & 50 & 1075 \\
Post-OPR 12-MW m & 773 & 230 & 200 & 1312 \\
Pre-OPR quality of life & 22.6 & 4.9 & 11.8 & 35.0 \\
Post-OPR quality of life & 28.7 & 5.1 & 16.1 & 38.4 \\
\hline
\end{tabular}

BMI: body mass index; FEV1: forced expiratory volume in one second; NP: nonpulmonary; 12-MW: 12 min walking distance; OPR: out-patient pulmonary rehabilitation. Quality of life refers to the total Chronic Respiratory Disease Quetionnaire score, with higher values indicating improved quality of life.

\section{Results}

One hundred and fifty eight patients (85 females and 73 males) completed OPR between November 1989 and March 1993. Pulmonary disease categories included: COPD (87\%), asthma (8\%), restrictive disease(2.5\%), and bronchiectasis $(2.5 \%)$. Patient characteristics are given in table 1. The mean FEV1 was $1.03 \pm 0.58 \mathrm{~L}$, which was $38 \pm 19 \%$ of predicted. Forty four percent had an FEV1 equal to or less than $0.75 \mathrm{~L}$. Twenty percent of the group were underweight and $41 \%$ were overweight. Twenty four percent used continuous, low-flow oxygen. Only $9 \%$ were employed.

The FEV1 was very weakly correlated with the preOPR 12-MW ( $\mathrm{r}=0.19 ; \mathrm{p}=0.03$ ), but not with the CRDQ total score or any of its dimensions. Correlations between the pre-OPR 12-MW and CRDQ are as follows: total score, $r=0.23(\mathrm{p}=0.01)$; dyspnoea, $\mathrm{r}=0.37(\mathrm{p}<0.0001)$; function, $r=0.20(\mathrm{p}=0.03)$; emotion, $\mathrm{r}=0.15(\mathrm{NS})$; and mastery, $\mathrm{r}=0.04$ (NS).

Nonpulmonary diagnoses averaged $2.0 \pm 1.6$ per patient. Common co-morbid conditions are listed in table 2 . Although hypertension and cardiac disease were most prevalent, 32 patients (20\%) had a history of cancer. Four patients had histories of two cancers. Primary sites for cancer included: breast (10 patients); lung (7); bowel (5); prostate (5); bladder (2); lymphoma (2); kidney (1); thyroid (1); larynx (1); uterus (1); skin (melanoma) (1).

The mean number of medications was 5.8 \pm 2.7 . Commonly used types of medications included: inhaled betaagonists $80 \%$; theophylline $66 \%$; oral corticosteroids $44 \%$; ipratropium $41 \%$; inhaled corticosteroids $34 \%$; diuretics $32 \%$; psychotropics and hypnotics $30 \%$; oral betaagonists $28 \%$; acid-peptic therapy $27 \%$; calcium channel blockers $17 \%$; and digoxin $13 \%$.

Table 2. - Common co-morbid conditions

\begin{tabular}{lc}
\hline Disorder & Frequency \\
& $\%$ \\
\hline Hypertension & 28 \\
Cardiac disease & 27 \\
Cancer & 20 \\
Acid/peptic disease & 22 \\
Psychiatric disorder & 17 \\
Congestive heart failure & 10 \\
Arthritis & 8 \\
\hline
\end{tabular}

Table 3. - Proportional hazards model indicating variables associated with increased mortality following OPR

\begin{tabular}{lrrr}
\hline Variable & $\mathrm{n}$ & $\chi^{2}$ & p-value \\
\hline Total group analysis $(\mathbf{n}=\mathbf{1 5 8})$ & & & \\
Low post-OPR 12-MW & 143 & 19.1 & $<0.0001$ \\
Elevated $\mathrm{Pa}_{\mathrm{a}, \mathrm{CO}_{2}}$ & 130 & 18.4 & $<0.0001$ \\
Low pre-OPR 12-MW & 149 & 14.6 & $<0.0001$ \\
Reduced $\mathrm{P}_{\mathrm{a}, \mathrm{O}_{2}}$ & 130 & 14.5 & $<0.0001$ \\
Low FEV & 147 & 14.3 & $<0.0001$ \\
Low BMI & 158 & 5.2 & 0.0230 \\
Increased number of medications & 158 & 5.0 & 0.0258 \\
Respiratory deaths $(\mathbf{n}=\mathbf{1 4 3})$ & & & \\
Low post-OPR 12-MW & 129 & 20.0 & $<0.0001$ \\
Low pre-OPR 12-MW & 134 & 15.2 & $<0.0001$ \\
Low FEV 1 & 132 & 14.5 & $<0.0001$ \\
High $P \mathrm{a}, \mathrm{CO}_{2}$ & 118 & 16.7 & $<0.0001$ \\
Low $P \mathrm{a}, \mathrm{O}_{2}$ & 118 & 14.0 & 0.0002 \\
Low BMI & 143 & 5.1 & 0.0243 \\
Increased number of medications & 143 & 5.0 & 0.0258 \\
Increased post-OPR CRDQ dyspnoea & 106 & 4.6 & 0.0329 \\
Nonrespiratory deaths $(\mathbf{n}=\mathbf{1 3 2})$ & & & \\
Low post-OPR 12-MW & 122 & 6.9 & 0.0087 \\
Low pre-OPR 12-MW & 127 & 4.1 & 0.0430 \\
Low FEV 1 & 124 & 4.7 & 0.0296 \\
High $P \mathrm{a}, \mathrm{CO}_{2}$ & 105 & 5.8 & 0.0160 \\
\hline
\end{tabular}

$\mathrm{Pa}_{\mathrm{a}} \mathrm{CO}_{2}:$ arterial carbon dioxide tension; $\mathrm{Pa}, \mathrm{O}_{2}:$ arterial oxygen tension; CRDQ: Chronic Respiratory Disease Questionnaire. For further abbreviations see legend to table 1.

The two primary outcome measures, which were recorded before and shortly following OPR, were the 12-MW and the CRDQ quality of life. The 12-MW increased by $17 \%$, from $659 \pm 223$ to $773 \pm 230 \mathrm{~m}(\mathrm{p}<0.0001)$, while the total CRDQ score increased by $27 \%$, from $22.6 \pm 4.9$ to $28.7 \pm 5.1$ over this period.

Forty three patients (27\%) died during the interval from completion of OPR to the time of data collection. The mean time from completion of OPR to death was $26 \pm 15$ months. The 3 year survival was $80 \%$. The principal cause of death was from respiratory disease in 26 patients and nonrespiratory disease in 17 patients. Nonrespiratory deaths were due to cardiac disease (7 patients), cancer (5), neurological disease (2), and gastrointestinal disease (1).

Significant predictors of survival are listed in table 3 . Three survival analyses are given: 1) for the entire group (all deaths); 2) for respiratory deaths (17 nonrespiratory deaths excluded); and 3) for nonrespiratory deaths (26 respiratory deaths excluded). In none of the three analyses were age, sex, pulmonary diagnosis category, total number of nonpulmonary diagnoses, or any of the comorbid conditions listed in table 2 significantly related to survival. In addition, pre- to post-OPR changes in the 12-MW and the CRDQ total score and its dimensions were not related to long-term prognosis.

For the analysis of the entire group, the post-OPR 12MW was the most influential predictor of survival. This was followed in decreasing order by $P \mathrm{a}, \mathrm{CO}_{2}$. pre-OPR 12-MW, $P \mathrm{a}, \mathrm{O}_{2}$, FEV1 long-term oxygen therapy (LTOT) requirement, $\mathrm{BMI}$, and number of medications. In the stepwise regression analysis, the $P \mathrm{a}, \mathrm{CO}_{2}$ was the only variable added after the post-OPR 12-MW. For illustrative purposes, the 36 month survival curves for high and low post-OPR 12-MW are depicted in figure 1 . 


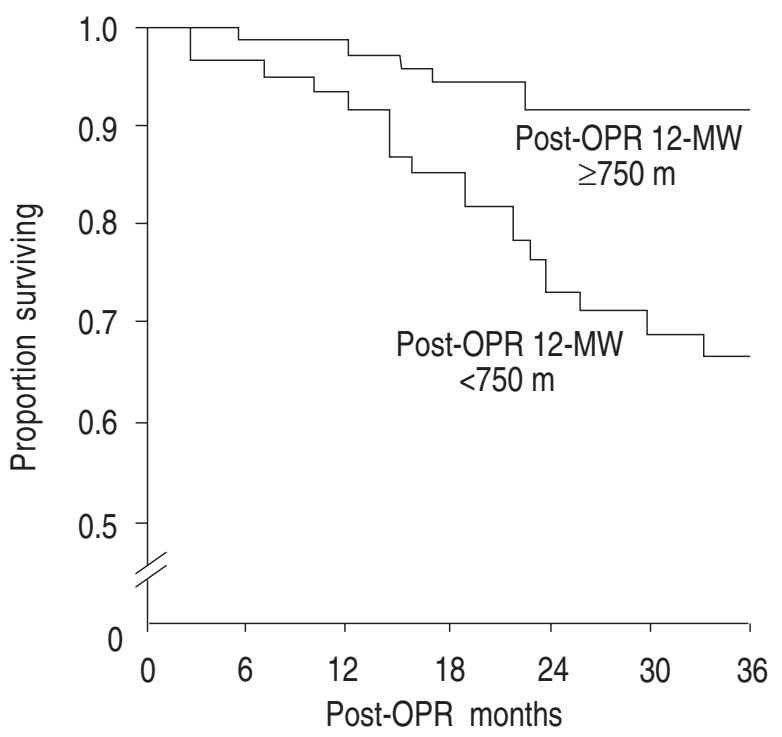

Fig. 1. - The post-OPR 12-MW and survival. Survival estimates for 36 months following OPR are depicted. Patients with a post-OPR 12MW of less than $750 \mathrm{~m}(\mathrm{n}=63)$ had a 3 year survival of $68 \%$, whilst those with a timed walking distance above this value $(n=80)$ had a 92\% survival $(\mathrm{p}<0.0001)$. OPR: out-patient pulmonary rehabilitation; 12-MW: 12 min walking distance.

For the analysis of respiratory deaths, significant predictor variables were similar to those for the entire group, with the post-OPR 12-MW as the most influential prognostic variable. In addition, post-OPR CRDQ dyspnoea was a significant, although relatively weak variable. In stepwise regression analysis, only the post-OPR 12-MW was significant.

For the analysis of nonrespiratory deaths, post-OPR $12-\mathrm{MW}$ was again the most influential prognostic variable, with pre-OPR 12-MW, FEV1, and $\mathrm{Pa}_{\mathrm{a}} \mathrm{CO}_{2}$ also significant. In stepwise regression analysis, as with deaths, only post-OPR 12-MW was significant.

\section{Discussion}

The purpose of this study was to evaluate which variables recorded at the time of OPR assessment were related to prognosis. Although the OPR patients were somewhat heterogeneous with respect to pulmonary diagnosis, the majority had COPD and all were symptomatic despite medical therapy. The mean FEV1 of $1.03 \mathrm{~L}$, which was $38 \%$ of predicted, reflects the advanced stage of ventilatory impairment in this group.

The $80 \% 3$ year survival following OPR is very similar to the $77 \% 3$ year survival in 985 COPD patients reported by the Intermittent Positive Pressure Breathing (IPPB) Trial Group in 1986 [4]. Although significant arterial hypoxaemia was excluded from the former study, the prebronchodilator FEV1 was almost identical to that of our group. In a more recent study, MAHLER et al. [12] found an $82 \% 2$ year survival in a group of 110 COPD patients. This group, however, had an FEV1 of $1.28 \mathrm{~L}$, which was somewhat less severe than in the present study.

Variables significantly related to increased mortality in our investigation included a short timed walking distance, hypoxaemia, increased $\mathrm{Pa}_{\mathrm{a}} \mathrm{CO}_{2}$, reduced FEV1, low BMI, and increased number of medications. Of note, other than for a weak association between increased CRDQ dyspnoea and respiratory mortality, quality of life was unrelated to long-term survival. Furthermore, although significant improvement was documented both in the 12-MW and quality of life following OPR, gains in these outcome areas were not related to subsequent survival.

This study underscores the importance of the timed walking distance as a predictor of long-term prognosis in advanced chronic lung disease. Thus, the 3 year survival was $92 \%$ for patients with a post-OPR 12-MW distance of $750 \mathrm{~m}$ or more, but only $68 \%$ for those below this value. The timed walking distance was a more influential prognostic variable than the FEV1, arterial blood gases, body weight, quality of life, co-morbidity, and oxygen or medication requirements. Even mortality from nonrespiratory causes was best predicted by a reduced timed walking distance.

As a measure of exercise performance in chronic lung disease, the timed walking distance is simple to administer, reproducible, responsive to therapeutic intervention, and very relevant to daily activities [9, 13, 14]. Performance on this exercise test correlates reasonably well with maximal performance on graded exercise testing $[15,16]$, and very well with general health status, as measured by the Sickness Impact Profile [17]. However, the weak association between timed walking distance and FEV1, observed in this study and others $[16,18]$, indicates that airflow obstruction is only one factor affecting this outcome measure. Nonrespiratory variables, such as cardiovascular fitness, nutritional status and muscular strength, probably affect performance on the timed walk [9, 13, 14], and may influence survival independent of the FEV1. Of note, the post-OPR 12-MW, which probably reflects maximal attainable performance better than its baseline value, was the stronger prognostic indicator.

In summary, the 12 minute walking distance was found to be a very strong predictor of long-term prognosis following out-patient pulmonary rehabilitation; patients with poorer performance on this exercise test had higher mortality. Thus, the timed walking distance is not only a useful outcome measure for pulmonary rehabilitation, it is an important indicator of prognosis in advanced respiratory disease.

\section{References}

1. Vale F, Reardon JZ, ZuWallack RL. The long-term benefits of out-patient pulmonary rehabilitation on exercise endurance and quality of life. Chest 1993; 103: 42-45.

2. Sahn SA, Nett LM, Petty TL. Ten year follow-up of a comprehensive rehabilitation program for severe COPD. Chest 1980; 77 (Suppl.): 311-314.

3 Bebout DE, Hodgkin JE, Zorn EG, et al. Clinical and physiological outcomes of a university-hospital pulmonary rehabilitation program. Respir Care 1983; 28: 1468-1473.

4. Anthonisen NR, Wright EC, Hodgkin JE, and the IPPB Trial Group. Prognosis in chronic obstructive pulmonary disease. Am Rev Respir Dis 1986; 133: 14-20.

5. Burrows B, Earle RH. Course and prognosis of chronic 
obstructive lung disease. N Engl J Med 1969; 280: 397-404.

6. Wilson DO, Rogers RM, Wright EC, Anthonisen NR. Body weight in chronic obstructive pulmonary disease. Am Rev Respir Dis 1989; 139: 1435-1438.

7. Kanner RE, Renzetti AD, Stanish WM, et al. Predictors of survival in subjects with chronic airflow limitation. Am J Med 1983; 74: 249-255.

8. Strom K. Survival of patients with chronic obstructive pulmonary disease receiving long-term domiciliary oxygen therapy. Am Rev Respir Dis 1993; 147: 585-591.

9. McGavin CR, Gupta SP, McHardy GJR. Twelve minute walking test for assessing disability in chronic bronchitis. $\mathrm{Br}$ Med $J$ 1976; 1: 822-823.

10. Guytt GH, Berman LB, Townsend M, Pugsley SO, Chambers LW. A measure of quality of life for clinical trials in chronic lung disease. Thorax 1987; 42: 773-778.

11. SAS Technical Report P-229. Chapter 19. The phreg procedure. Cary, NC, 1992; pp. 443-480.

12. Mahler DA, Tomlinson D, Olmstead EM, Tosteson ANA, O'Connor GT. Changes is dyspnoea, health status, and lung function in chronic airway disease. Am J Respir Crit Care Med 1995; 151: 61-65.
13. Guyatt GH, Thompson PJ, Berman LB, et al. How should we measure function in patients with chronic heart and lung disease? J Chron Dis 1985; 38: 517-524.

14. Cockcroft AE, Saunders MJ, Berry G. Randomized controlled trial of rehabilitation in chronic respiratory disability. Thorax 1981; 36: 200-203.

15. Bernstein ML, Despars JA, Singh NP, Avalos K, Stansbury DW, Light RW. Reanalysis of the 12 minute walk in patients with chronic obstructive pulmonary disease. Chest 1994; 105: 163-167.

16. Swinburn CR, Wakefield JM, Jones PW. Performance, ventilation, and oxygen consumption in three different types of exercise test in patients with chronic obstructive lung disease. Thorax 1985; 40: 581-586.

17. Jones PW, Baveystock CM, Littlejohns P. Relationships between general health measured with the sickness impact profile and respiratory symptoms, physiological measures, and mood in patients with chronic airflow limitation. Am Rev Respir Dis 1989; 140: 1538-1543.

18. Mahler DA, Weinberg DH, Wells CK, Feinstein AR. The measurement of dyspnoea: contents, interobserver agreement, and physiologic correlates of two new clinical indexes. Chest 1984; 85: 751-758. 